\title{
Odd-parity Nucleon Eigenstates in Full QCD
} \author{
University of Adelaide, SA 5005, Australia. \\ E-mail: md.mahbub@adelaide.edu.au, \\ waseem.kamleh@adelaide.edu.au, \\ derek. leinweber@adelaide.edu.au, \\ peter.moran@alumni.adelaide.edu.au, \\ anthony.williamseadelaide.edu.au
}

M. S. Mahbub*, W. Kamleh, D. B. Leinweber, P. J. Moran, A. G. Williams Special Research Centre for the Subatomic Structure of Matter, School of Chemistry \& Physics,

\begin{abstract}
The results for the odd-parity nucleon spectrum at light quark masses are presented. The $2+1$ flavor gauge configurations generated by the PACS-CS collaboration are used and the analysis is performed at the lightest pion mas of $156 \mathrm{MeV}$. The energy eigenstates are tracked by following the evolution of the eigenvector as the quark mass changes. The two lowest-energy odd-parity states revealed are found to be in accord with the physical $N \frac{1}{2}^{-}$spectrum of Nature.
\end{abstract}

The 30th International Symposium on Lattice Field Theory

June 24 - 29, 2012

Cairns, Australia

${ }^{*}$ Speaker. 


\section{Introduction}

In hadron spectroscopy, the experimentally observed mass of the lowest positive-parity excitation of the nucleon is known as the Roper resonance $N \frac{1}{2}^{+}(1440) \mathrm{P}_{11}$. It is surprisingly low compared to the lowest-lying negative-parity partner $N \frac{1}{2}^{-}$(1535) $\mathrm{S}_{11}$, and this has been puzzling researchers since its discovery in the 1960's. This phenomenon is not observed in constituent or valence quark models where the lowest-lying odd-parity state occurs naturally below the first $J^{P}=\frac{1}{2}^{+}$ excitation.

Lattice QCD is the only currently known ab-initio or first-principles approach to the fundamental quantum field theory governing the properties of hadrons, Quantum Chromodynamics (QCD). The ground-state hadron spectrum of QCD is well understood in this non-perturbative approach. However, a determination of the excited state energy spectrum is still in the process of being revealed. Some of the problems in exploring the QCD excitations include: finding an optimum set of operator basis that can couple strongly to the states of interest; obtaining suitable techniques in isolating the superposed states; and controlling uncertainties so that extracted results are reliable and can be compared with the existing experimental data.

The low-lying $N \frac{1}{2}^{-}$energy states provide an interesting platform for Lattice QCD studies of spectroscopy. The experimentally observed nearly-degenerate $S_{11}(1535)$ and (1650) states are in agreement with the $\mathrm{SU}(6)$ quark-model predictions. Complementing these results by exploring the low-lying $N \frac{1}{2}^{-}$energy states and their structure from a first principles approach is important.

Here, we present results for the lowest-lying $N_{1^{-}}{ }^{-}$channel including the dynamical fermion loops of full QCD, using the PACS-CS $2+1$ flavor gauge field configurations [1]. We use the wellestablished variational method $[2,3]$ in this investigation using smearing at the fermion sources and sinks. Some recent full QCD results can be seen in Refs. [4, 5, 6, 7, 8, 9].

\section{Variational Method}

The two-point correlation-function matrix for $\vec{p}=0$ can be written as

$$
G_{i j}^{ \pm}(t)=\sum_{\vec{x}} \operatorname{Tr}_{\mathrm{sp}}\left\{\Gamma_{ \pm}\left\langle\Omega\left|\chi_{i}(x) \bar{\chi}_{j}(0)\right| \Omega\right\rangle\right\}
$$

where Dirac indices are implicit, $\bar{\chi}_{j}$ and $\chi_{i}$ are creation and annihilation operators and $\Gamma_{ \pm}=\left(\gamma_{0} \pm\right.$ 1) $/ 2$ projects the parity of the eigenstates. A linear superposition of interpolators $\bar{\phi}^{\alpha}=\sum_{j} \bar{\chi}_{j} u_{j}^{\alpha}$ creating state $\alpha$ with mass $m_{\alpha}$ provides the relationship

$$
G_{i j}\left(t_{0}+\triangle t\right) u_{j}^{\alpha}=e^{-m_{\alpha} \triangle t} G_{i j}\left(t_{0}\right) u_{j}^{\alpha},
$$

from which right and left eigenvalue equations are obtained

$$
\begin{aligned}
& {\left[\left(G\left(t_{0}\right)\right)^{-1} G\left(t_{0}+\triangle t\right)\right]_{i j} u_{j}^{\alpha}=c^{\alpha} u_{i}^{\alpha},} \\
& v_{i}^{\alpha}\left[G\left(t_{0}+\triangle t\right)\left(G\left(t_{0}\right)\right)^{-1}\right]_{i j}=c^{\alpha} v_{j}^{\alpha},
\end{aligned}
$$

with $c^{\alpha}=e^{-m_{\alpha} \triangle t}$. The vectors $u_{j}^{\alpha}$ and $v_{i}^{\alpha}$ diagonalize the correlation matrix at times $t_{0}$ and $t_{0}+\triangle t$ making the projected correlation matrix, $G_{ \pm}^{\alpha} \equiv v_{i}^{\alpha} G_{i j}^{ \pm}(t) u_{j}^{\alpha}$, which is then analyzed to obtain masses of energy-states. Details of our method can be found in Ref. [10]. 
We can insert $G\left(t_{0}\right)^{-\frac{1}{2}} G\left(t_{0}\right)^{\frac{1}{2}}$, in Eq. (2.3) and multiply by $G\left(t_{0}\right)^{\frac{1}{2}}$ from the left to give

$$
G\left(t_{0}\right)^{-\frac{1}{2}} G\left(t_{0}+\triangle t\right) G\left(t_{0}\right)^{-\frac{1}{2}} w^{\alpha}=c^{\alpha} w^{\alpha},
$$

where, $w^{\alpha}=G\left(t_{0}\right)^{\frac{1}{2}} u^{\alpha}$. Here, $\left[G\left(t_{0}\right)^{-\frac{1}{2}} G\left(t_{0}+\triangle t\right) G\left(t_{0}\right)^{-\frac{1}{2}}\right]$ is a real symmetric matrix, with orthogonal eigenvectors $w^{\alpha}$. The vector $u^{\alpha}$ may be recovered from the $w^{\alpha}$ via $u^{\alpha}=G\left(t_{0}\right)^{-\frac{1}{2}} w^{\alpha}$.

\section{Results}

Our analysis is performed on the PACS-CS $2+1$ flavor dynamical-fermion configurations [1] made available through the International Lattice Data Grid (ILDG) [11]. Details of our propagator parameters can be found in Ref. [12].

Results for the lowest $N \frac{1}{2}^{-}$energy state from dynamical QCD are presented in Fig. 1 and compared to the quenched results [13] for the same state. The scale is set via the Sommer parameter [14]. These results are from a simple correlation matrix analysis using the four different smearings of Ref. [12] and the scalar di-quark interpolator $\chi_{1}$.

As expected, the dynamical and quenched QCD results are in agreement in the heavy-quark mass region. However, in the light quark-mass regime the results are significantly different. Here the effects of the light sea quarks become important. Only the dynamical results (i.e., full QCD) approach the physical value of the lowest $N \frac{1}{2}^{-}$(1535) state. This provides strong evidence for the non-trivial role of light sea-quarks to the structure of the $N \frac{1}{2}^{-}$spectrum.

The experimentally observed nearby second energy state, $S_{11}(1650)$, is nearly degenerate with the $S_{11}$ (1535) state. Therefore, investigating the (1650) MeV state is highly desirable from the firstprinciples approach. Here we extend our analysis incorporating different spin-flavor combinations of interpolators $\chi_{2}$ and $\chi_{4}$ [12].

The results of an $8 \times 8$ correlation matrix analysis using $\chi_{1}$ and $\chi_{2}$ interpolators with four levels of smearing are presented in Fig. 2. The flow of the lowest two energy states towards the physical values is in accord with the physical spectrum. The results at the two heaviest pion masses sit close to the scattering S-wave $N+\pi$ threshold. However, in the light quark-mass region these states move above the threshold. The situation is similar for the second pair of states, where they sit close to the scattering p-wave $E_{N}+E_{\pi}+M_{\pi}$ and $E_{\pi}+E_{\pi}+M_{N}$ thresholds. Also their approach to the $2090 \mathrm{MeV}$ state is interesting.

Now we discuss how we assign symbols to each of the energy levels observed at a particular quark mass of Fig. 2. In doing so, it is necessary to track the evolution of the states from one quark mass to the next. We have done this through a consideration of the evolution of the eigenvectors as the quark mass is changed.

Consider $M$ interpolating fields making an $M \times M$ parity-projected correlation matrix $G(t)$ and its associated symmetric generalized eigenvalue equation of Eq. (2.5). Using the normalization $\sum_{i}^{M}\left|w_{i}^{\alpha}\right|^{2}=1$, the quantity $\vec{w}^{\alpha}\left(m_{q}\right) \cdot \vec{w}^{\beta}\left(m_{q}\right)=\delta_{\alpha \beta}$. This feature enables the use of the generalized measure

$$
\mathscr{W}^{\alpha \beta}\left(m_{q}, m_{q^{\prime}}\right)=\vec{w}^{\alpha}\left(m_{q}\right) \cdot \vec{w}^{\beta}\left(m_{q^{\prime}}\right)
$$




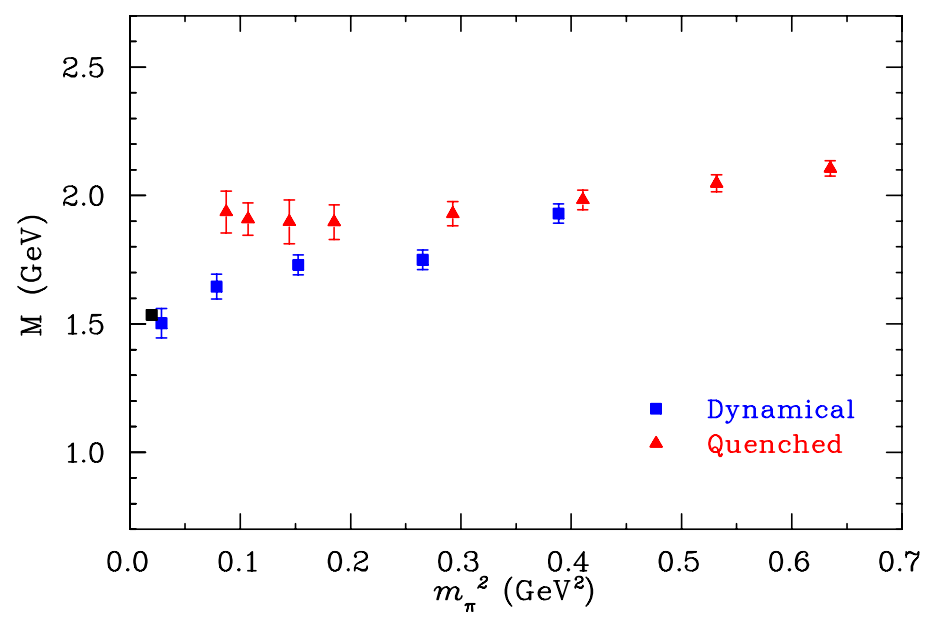

Figure 1: (Color online). Dynamical and quenched [13] QCD results for the lowest $N \frac{1}{2}^{-}$energy-state using the scalar-diquark interpolator, $\chi_{1}$. Sea-quarks loops play a significant role in the $N \frac{1}{2}^{-}$dynamics.

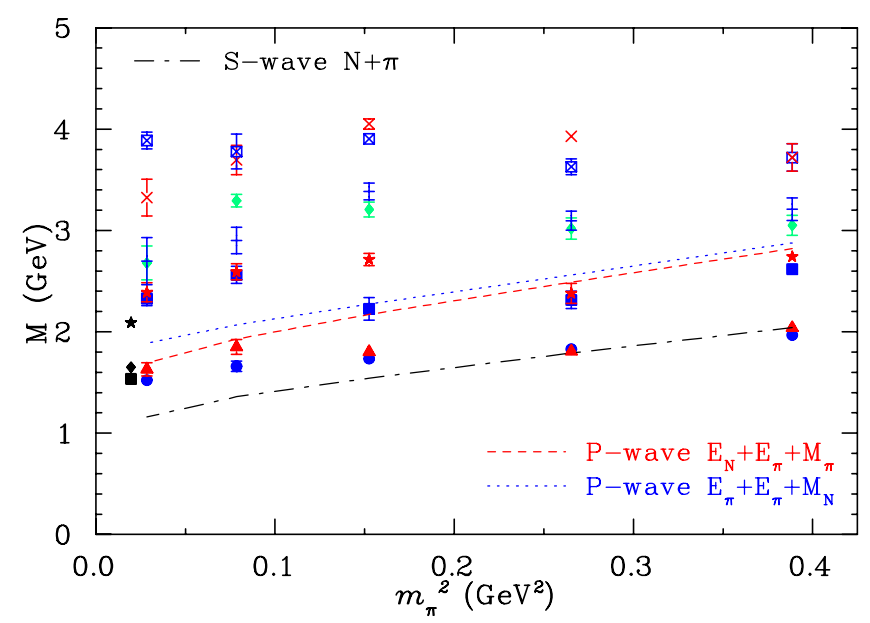

Figure 2: (Color online). $N \frac{1}{2}^{-}$eigenstate energies from an $8 \times 8$ correlation matrix analysis of $\chi_{1}$ and $\chi_{2}$ interpolators. The physical $N \frac{1}{2}^{-}$spectrum [15] is shown at the far left.

to identify the states most closely related as we move from quark mass $m_{q}$ to an adjacent quark mass $m_{q^{\prime}}$. The state numbers $\alpha$ and $\beta$ are assigned in order of increasing projected eigenstate energy at the quark masses $m_{q}$ and $m_{q^{\prime}}$ respectively. Typical results for this generalized measure of eigenvector overlap are presented in Table 1.

For each value of state index $\alpha$ there is only one value of $\beta$ where the magnitude of the entry is significantly larger than all others and approaching unity. The most relevant entries for consideration are the immediate neighbors of $\alpha$ where a crossing of the eigenvectors moves the largest entry off the diagonal. It is evident that the tracking of eigenvectors is robust with $\vec{w}^{\alpha}\left(m_{q}\right)$. $\vec{w}^{\beta}\left(m_{q^{\prime}}\right) \sim 1$. The components of the eigenvector $w^{\alpha}$ are presented in Fig. 3.

This measure provides a clear identification of how states in the spectrum at quark mass $m_{q}$ are associated with states at the next value of quark mass, $m_{q^{\prime}}$. For example, the results of Table 1 indicate the first four states at $m_{q^{\prime}}$ appear with the same ordering in the spectrum as observed at 
Table 1: The scalar product $\vec{w}^{\alpha}\left(m_{q}\right) \cdot \vec{w}^{\beta}\left(m_{q^{\prime}}\right)$ for $\kappa=0.13754\left(m_{\pi}=413 \mathrm{MeV}\right)$ and $\kappa^{\prime}=0.13770\left(m_{\pi}=\right.$ $293 \mathrm{MeV}$ ) for an $8 \times 8$ correlation matrix of $\chi_{1}$ and $\chi_{2}$ with four different levels of smearing. State numbers $\alpha$ and $\beta$ correspond to row and column number, respectively.

\begin{tabular}{cccccccc}
\hline $\mathbf{0 . 9 1}$ & 0.40 & 0.02 & 0.02 & 0.01 & -0.05 & 0.00 & 0.00 \\
0.40 & $\mathbf{- 0 . 9 1}$ & 0.00 & 0.01 & -0.02 & 0.01 & -0.01 & 0.00 \\
-0.01 & -0.01 & $\mathbf{0 . 9 6}$ & -0.27 & 0.01 & -0.01 & 0.00 & 0.02 \\
-0.03 & 0.00 & 0.27 & $\mathbf{0 . 9 6}$ & 0.01 & 0.01 & 0.02 & 0.00 \\
0.04 & 0.03 & 0.01 & -0.01 & -0.22 & $\mathbf{0 . 9 7}$ & 0.02 & 0.01 \\
0.01 & -0.01 & -0.01 & -0.01 & $\mathbf{0 . 9 8}$ & 0.22 & 0.04 & 0.00 \\
0.00 & 0.00 & -0.02 & 0.01 & 0.01 & -0.01 & -0.12 & $\mathbf{0 . 9 9}$ \\
0.01 & -0.01 & 0.00 & -0.02 & -0.04 & -0.03 & $\mathbf{0 . 9 9}$ & 0.12 \\
\hline
\end{tabular}

$m_{q}$, the fifth state at $m_{q^{\prime}}$ is associated with the sixth state at $m_{q}$ and vice versa and similarly for the seventh and eighth states. We note that while the central values of the energies have changed order, the error bars are sufficiently large that one cannot conclude that an avoided energy level crossing has taken place in moving from quark mass $m_{q}$ to $m_{q^{\prime}}$.

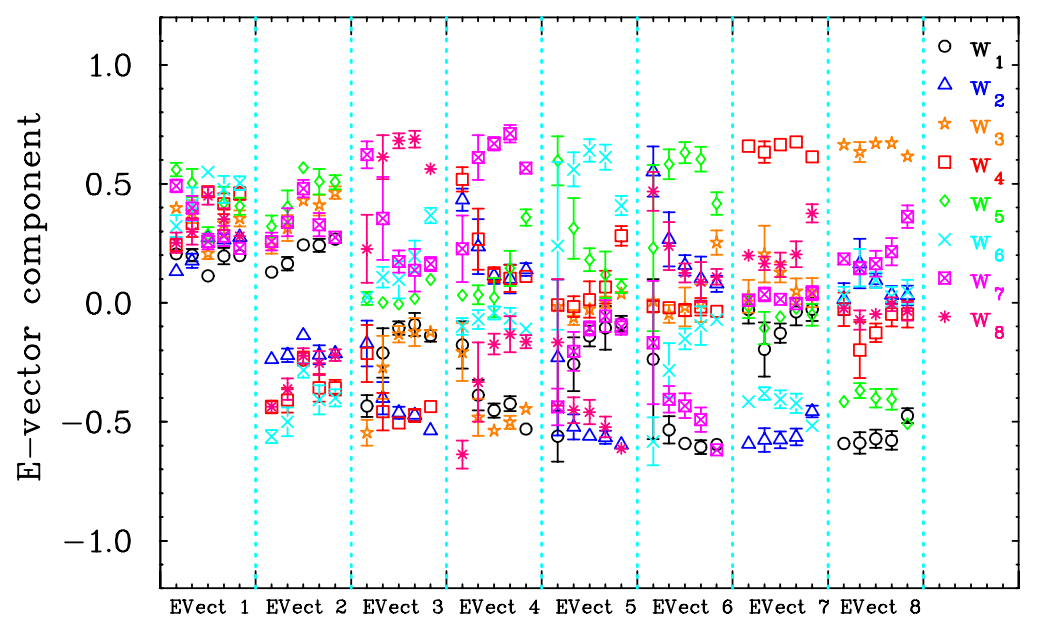

Figure 3: (Color online). The components of the eigenvector $w^{\alpha}$. The states are labeled by the eigenvector (EVect) number with the ordering as provided in Fig. 2 at the heaviest quark mass. For each EVect, the components of $w$ are plotted from left to right in order of increasing quark mass. In the legend, $\left(w_{1}, w_{2}\right)$, $\left(w_{3}, w_{4}\right),\left(w_{5}, w_{6}\right)$ and $\left(w_{7}, w_{8}\right)$ correspond to the smearing-sweep levels of 16, 35, 100 and 200 respectively. Odd values are for $\chi_{1}$ and even for $\chi_{2}$.

The components of the eigenvector $u^{\alpha}$, providing the amplitude for each interpolating field at the source for creating the state $\alpha$, are provided in Fig. 4. A non-trivial contribution from both the $\chi_{1}$ and $\chi_{2}$ interpolators for the lowest two energy-states is evident. The scalar-diquark interpolator $\chi_{1}$ dominates the lowest energy-state. On the other hand, both $\chi_{1}$ and $\chi_{2}$ interpolators have large contributions to the second energy state where their strengths appear with opposite signs. The eigenvector components typically display a slow evolution as the quark mass is changed.

The two lowest-lying $N \frac{1}{2}^{-}$states are presented in Fig. 5 in comparison with the $S$-wave scattering threshold. These lattice results are in agreement with the physical values. Although both these 


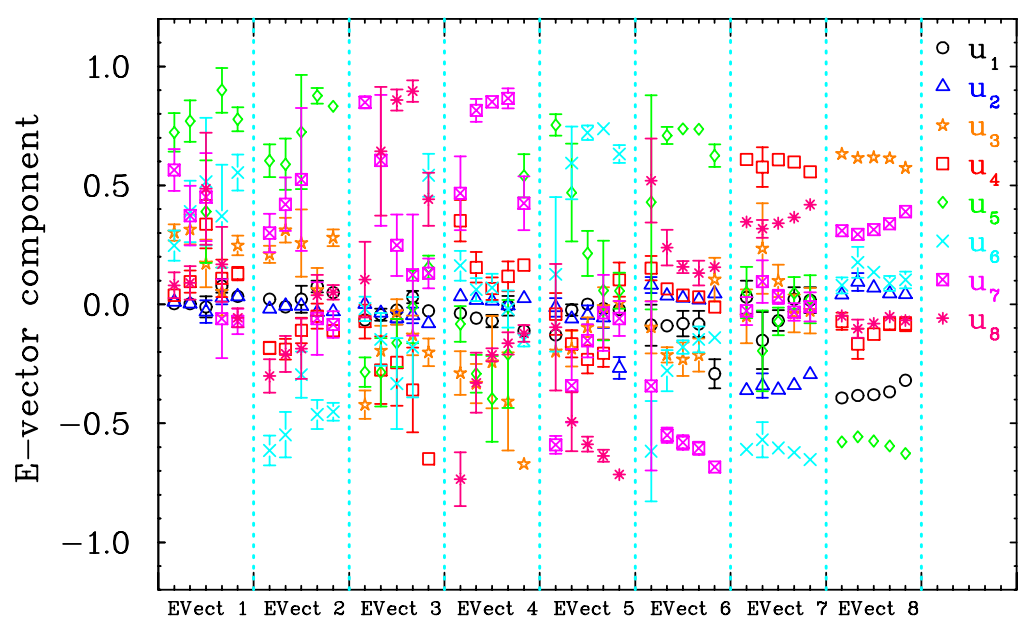

Figure 4: (Color online). The components of the eigenvector $u^{\alpha}$. For each EVect, the components of $u$ are plotted from left to right in order of increasing quark mass. In the legend, $\left(u_{1}, u_{2}\right),\left(u_{3}, u_{4}\right),\left(u_{5}, u_{6}\right)$ and $\left(u_{7}, u_{8}\right)$ correspond to the smearing-sweep levels of 16, 35, 100 and 200 respectively. Odd numbers in the subscripts correspond to the contribution from the $\chi_{1}$ interpolator, whereas, even numbers correspond to $\chi_{2}$.

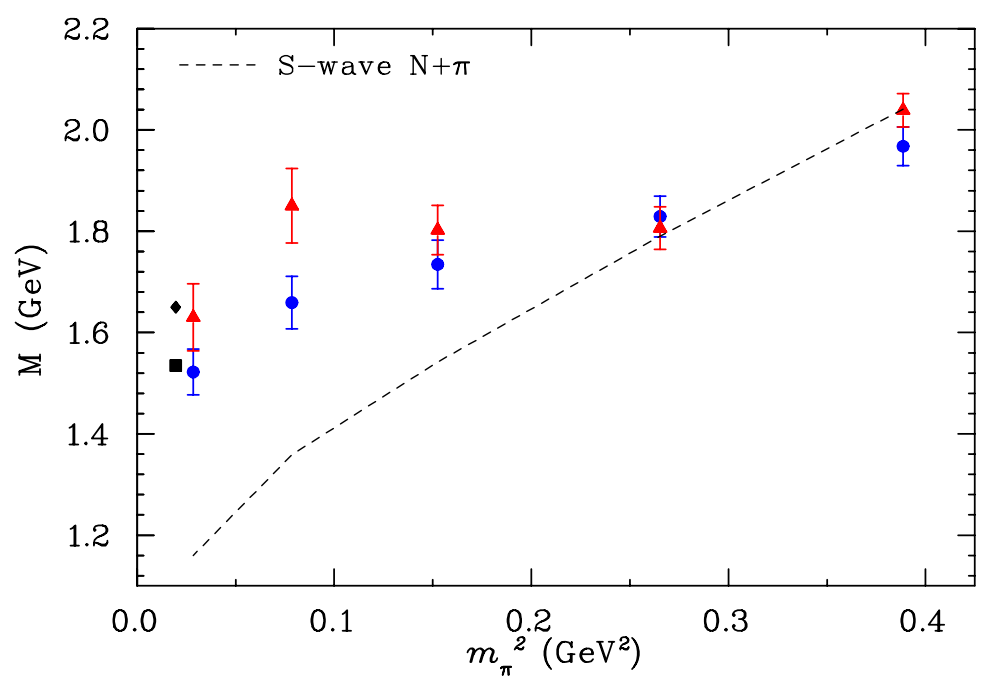

Figure 5: (Color online). The quark mass dependence of the lowest two $N \frac{1}{2}^{-}$states.

low-lying states are quite similar at the two heaviest quark masses, their approach to the physical values in the light quark-mass region are different, in particular, a significant chiral curvature for the second state is evident. Future studies will seek to observe the multi-particle scattering states and determine the resonance parameters.

\section{Acknowledgments}

We thank PACS-CS Collaboration for making these $2+1$ flavor configurations available and the ILDG for creating the opportunity, tools and formalism for sharing these configurations. This research was undertaken on the NCI National Facility in Canberra, Australia, which is supported by the Australian Commonwealth Government. We also acknowledge eResearch SA for grants of 
supercomputing time which have enabled this project. This research is supported by the Australian Research Council.

\section{References}

[1] S. Aoki, et al., Phys. Rev. D 79 (2009) 034503. arXiv:0807.1661.

[2] C. Michael, Nucl. Phys. B259 (1985) 58.

[3] M. Luscher, U. Wolff, Nucl. Phys. B339 (1990) 222-252.

[4] J. M. Bulava, et al., Phys. Rev. D79 (2009) 034505. arXiv:0901.0027, doi:10.1103/PhysRevD.79.034505.

[5] J. Bulava, et al., Phys. Rev. D82 (2010) 014507. arXiv:1004.5072, doi:10.1103/PhysRevD.82.014507.

[6] G. P. Engel,C. B. Lang,M. Limmer,D. Mohler, A. Schafer, Phys. Rev. D82 (2010) 034505. arXiv:1005.1748, doi:10.1103/PhysRevD.82.034505.

[7] R. G. Edwards,J. J. Dudek,D. G. Richards, S. J. Wallace, Phys. Rev. D84 (2011) 074508. arXiv:1104.5152, doi:10.1103/PhysRevD.84.074508.

[8] M. S. Mahbub,W. Kamleh,D. B. Leinweber,P. J. Moran, A. G. Williams, Phys. Lett. B707 (2012) 389-393. arXiv:1011.5724.

[9] B. J. Menadue,W. Kamleh,D. B. Leinweber, M. S. Mahbub, Phys. Rev. Lett. 108 (2012) 112001. arXiv:1109.6716, doi:10.1103/PhysRevLett.108.112001.

[10] M. S. Mahbub,A. O. Cais,W. Kamleh,D. B. Leinweber, A. G. Williams, Phys. Rev. D82 (2010) 094504. arXiv:1004.5455.

[11] M. G. Beckett, et al., Comput. Phys. Commun. 182 (2011) 1208-1214. arXiv:0910.1692, doi:10.1016/j.cpc.2011.01.027.

[12] M. S. Mahbub,W. Kamleh,D. B. Leinweber,P. J. Moran, A. G. WilliamsarXiv:1209.0240.

[13] M. S. Mahbub,W. Kamleh,D. B. Leinweber,A. O. Cais, A. G. Williams, Phys. Lett. B693 (2010) 351-357. arXiv:1007.4871, doi:10.1016/j.physletb.2010.08.049.

[14] R. Sommer, Nucl. Phys. B411 (1994) 839-854. arXiv:hep-lat/9310022, doi:10.1016/0550-3213(94)90473-1.

[15] K. Nakamura, et al., J. Phys. G37 (2010) 075021. doi:10.1088/0954-3899/37/7A/075021. 